\title{
Diversity of Aphid Pests (Homoptera: Aphididae) and their Natural Bio-Control Agents in Vegetable Crop Ecosystems of Jammu \& Kashmir, India
}

\author{
Deen Mohd. Bhat ${ }^{1 *}$, Sajad Ahmad Khan², Fayaz Ahmad Ahanger ${ }^{3}$ \\ and Muzafar Ahmad Sheikh ${ }^{1}$
}

\author{
${ }^{1}$ Department of Zoology, Govt. Degree College Ganderbal, J\&K-191201, India \\ ${ }^{2}$ Department of Zoology, Baba Gulam Shah Badshah University, Rajouri, J\&K-India \\ ${ }^{3}$ Department of Zoology, Govt. College for Women, M. A. Road, Srinagar, J\&K-190006, India
}

*Corresponding author

\begin{abstract}
A B S T R A C T
The aphids (Homoptera: Aphididae) are one of major pests of agriculturally important crops, including vegetables, across the world. They suck plant sap, cause leaf curling, yellowing and stunted growth of the host plants. They also act as vectors of many viral diseases in plants. In order to explore aphid pests and their naturally occurring bio-control agents (parasitoids and predators), an extensive field survey was conducted in different vegetable ecosystems of the Kashmir valley during the years from 2014-2019. Moreover, a through literature survey pertaining to aphids, reported from Jammu and Kashmir, was also done. Accordingly, based on these studies, it was concluded that as many as 18 species of aphids belonging to 12 genera infest as many as 26 species and 30 varieties of vegetable crops in Jammu and Kashmir (J\&K). It was also concluded that at least 29 species of insect predators and 17 species of parasitoids occur naturally to suppress these aphid pest species in vegetable ecosystems in J\&K. In sum, through this study, a comprehensive account of aphids and their natural enemies with reference to vegetable crops in $\mathrm{J} \& \mathrm{~K}$ is presented. This work will be useful for the studies concerning the devising of strategies for Integrated Control Program of aphids, especially through utilization of natural bio-control agents.
\end{abstract}

\section{Introduction}

Aphids or 'plant lice' are minute pear-shaped, soft-bodied organisms. They belong to Class Insecta, order Homoptera and family Aphididae. They may be winged (alate) or wingless (apterous). Their life cycle is completed through egg, nymphal and adult stages. Apart from sexual reproduction, they can also reproduce through a process of parthenogenesis (Dixon, 1987 and 1998). Aphids are an important group of plant insect pests having a high biotic potential as reflected by their parthenogenicity, viviparity 
and fast development. They are found to inflict direct (sucking) and indirect (transmission of viruses and honeydew secretion) damage to different types of vegetations and are considered the most potent and worldwide enemies of many crops (Blackmon and Eastop, 2007)

Aphids are an extremely successful group which occurs throughout the world, with the greatest number of species in the temperate regions (Blackmon and Eastop, 2007). Out of 4702 aphid species so far known world over (Remaudiere and Remaudiere, 1997), about 1015 species occur in Oriental region (Agarwala and Gosh, 1984 ), among which, about 750 species belonging to 208 genera represent Indian Aphididae (Gosh and Gosh, 2006).

In Jammu and Kashmir region (India), more than 31 species of aphids have been reported on wide range of host-crop species of agricultural, horticultural and forest ecosystems, with varying degree of host specificity (Bhagat, 2012). Among these aphids, some species had earlier been reported on vegetable crops and the significant studies in this regard have been conducted by Ahmad and Bhat (1986); Bhagat (1986, 2012); Zaz (2001); Pandey et al., (2006); Bhat (2008, 2017); Khan et al., (2009, 2017); Bhat et al., (2011); Bhat and Bhagat (2017) and Bhat and Ahanger (2018).

Some natural enemies (parasitoids/predators) had earlier been reported on aphid pests on some vegetable crops in $\mathrm{J} \& \mathrm{~K}$ region and the important studies in this connection were done by Ahmad and Bhat (1986); Bhagat and Lone (1984); Bhagat \& Masoodi (1986); Bali (1987); Bhagat \& Matta (2002); Bhat (2008a) ; Khan et al., (2009, 2017); Shah and Khan (2013); Bhat (2017) and Bhat \& Bhagat (2017). Similarly, some parasitoid species were earlier recorded to suppress the aphid population in vegetable ecosystems in $\mathrm{J} \& \mathrm{~K}$ region and the key researchs in this regard were conducted by Rao et al., (1969); Shujauddin (1973); Stary and Bhagat (1978); Takada \& Rishi (1980); Bhagat (1982 a, 1982b, 1986, 2008); Bhagat \& Ahmad (1991); Bhat (2008a), Khan et al., (2017) and Bhat et al., (2017).

However, all the aforementioned studies pertaining to aphids and their natural enemies in $\mathrm{J} \& \mathrm{~K}$ were scattered in different journals and, hitherto, there was no consolidated checklist available concerning aphids and their natural enemies on vegetable crops in $\mathrm{J} \& \mathrm{~K}$. Therefore, in sum, this paper by way of adding new information and consolidating the previous works pertaining to aphids and their natural enemies, gives a clearer picture regarding this subject area. Accordingly, a comprehensive account of the systematic position, host-crop range and natural biocontrol agents of 18 species of aphids along with their 45 species of natural enemies (parasitoids and predators) in $\mathrm{J} \& \mathrm{~K}$ is provided through this work. This study will be helpful in future for understanding of biodiversity of aphis and their natural enemies, particularly from this region. Moreover it would also be useful for the studies concerning the devising of strategies of integrated control program for aphids, especially through utilization of natural enemies (as bio-control agents).

\section{Materials and Methods}

\section{Literature survey and preparation of compendium/checklist of aphid pests}

A comprehensive literature survey of all the available published works/ research papers etc., published from time to time pertaining to aphid pest and their natural bio-control agents (parasites and predators) in $\mathrm{J} \& \mathrm{~K}$ regions, was done in order to collect the information. Besides consulting previous published papers, 
the other vital e-resources and abstracting services, particularly, CAB, NISCAIR, AGRI, Biological abstracts, etc., were also consulted for obtaining the required information.

\section{Field and laboratory studies}

The data presented in this paper is also based on extensive field surveys of aphids and their natural bio-control agents(parasites and predators), in different vegetable farms, floating gardens, kitchen gardens etc in different localities of $\mathrm{J} \& \mathrm{~K}$ region, conducted by the present authors over the years from 2007-2019, appended as under:-

\section{Study area (Fig. 62)}

Regular fortnightly random and extensive field surveys were conducted at 7study sites across the length and breadth of Kashmir Valley viz. Danderkhah in District Srinagar $\left(34.0687^{\circ} \mathrm{N}, 74.7783^{\circ} \mathrm{E}\right)$, Zazuna in District Ganderbal $\left(34.2301^{\circ} \mathrm{N}, 74.6854^{\circ} \mathrm{E}\right)$, Bugam in District Budgam $\left(33.6911^{\circ} \mathrm{N}, 75.0231^{\circ} \mathrm{E}\right)$, Bangidar in District Anantnag $\left(33.7265^{\circ} \mathrm{N}\right.$, $\left.75.1443^{\circ} \mathrm{E}\right)$, Chaklu in District Baramulla $\left(34.2004^{\circ} \mathrm{N}, 74.3969^{\circ} \mathrm{E}\right)$, Sumlar in District Bandipora $\left(34.4111^{\circ} \mathrm{N}, 74.7235^{\circ} \mathrm{E}\right)$ and Murran in District Pulwama $\left(33.8664^{\circ} \mathrm{N}\right.$, $\left.74.8639^{\circ} \mathrm{E}\right)$.

\section{Sampling method and rearing}

The vegetable farms/ vegetable gardens/ kitchen gardens in above cited study areas were surveyed. Three fields at every site were selected randomly and sampling procedure was based on standardized sampling techniques. The field data regarding aphid pests, their host-plant, predators (natural enemy) was recorded in field diary. The immature stages (nymphs) of aphids were taken to laboratory, along with predatory larvae, if any and were reared on the host plant in glass / plastic jars for the development of their adults and for the recovery of their parasitoids. The adults of aphids, their parasitoids and predators which emerged after rearing were identified and preserved.

\section{Presentation of data in the form of checklist}

The necessary data, pertaining to aphid is documented and presented in this paper in the form of checklist. The aphid species are listed sub family-wise \& systematically, with details given on their host-plant range (followed by references), insect predators (family wise and followed by references) and parasitoids (followed by references).

\section{Results and Discussion}

The results obtained during this work are highlighted through a below mentioned checklist, well supported by field photography (Fig. 1-61) and table 1. The checklist reveals that as many as 18 species of aphids (See Section b) belonging to12 genera under 2 subfamilies (Aphidiinae and Lachinae) of family Aphididae (Order: Homoptera) infest at least 26 species and 30 varieties of vegetable crops (See Section a) in $\mathrm{J} \& \mathrm{~K}$.

The checklist also reveals that at least 29 species of insect predators (natural enemies) (See Section c), belonging to 25 genera under 5 families (Anthocoridae, Coccinellidae, Ceccidomyiidae, Syrphidae, Chrysoperlidae) under 4 Insect orders (Coleptera, Hemiptera, Diptera Neruoptera) commonly occur and feed on afore-mentioned aphid species. Apart from this, 17 species of parastioids (Hymenoptera: Braconidae: aphidiinae) (See Section d), belonging to 7 genera which parasitize these aphids on vegetable crops, have been documented in this checklist. 
Summary of vegetable plant host-range of aphid pests in $\mathrm{J} \& \mathrm{~K}$

As highlighted above, 26 species and 30 varieties of vegetable crops (both cultivated and wild) have been found infested by Aphid pests. These vegetable plants are: Brassica napus (Linn.) (= Brassica campestris L.), Brassica juncea Linn., Brassica napus (Linn.), Brassica oleracea (Linn.)- Brassica oleracea var. acephella (kale), Brassica o. var. botrytis (Cauliflower), B. oleracea var. capitata (cabbage), B. o.var. kashmiriana, B. o. var. gongylodes (knolkhol), Brassica rapa L. (turnip), Capcicum anпиum L. (capsicum), Coriandrum sativum $\mathrm{L}$. (Corriander), Cucumis sativus (Linn.) (Cucumber), Cucurbita maxima Duchensc (large pumpkin), Cucurbita pepo L. (Marrow), Daucus carota L. (carrot), Dolichos lablab L. (Lablab bean), Helianthus tuberosus L. (artichoke), Hibiscus esculentus L (Okra), Lagenaria siceraria (Molina) (bottle gourd), Lycopersicon esculentum Mill (tomato), Phaseolus coccineus (Linn.) (runner bean), Phaseolus vulgaris (common bean/French beans), Pisum sativum Linn. (pea), Raphanus sativus L. (radish), Rumex acetosa (Linn.), Rumex nepalensis Spreng Rumix sp. (rumex), Solanum tuberosum (Linn.), Solanum melongena L., Taraxacum officinale (L.).

Summary of aphid pest species of vegetable crops in $\mathbf{J} \& \mathbf{K}$

The 18 species of Aphids (Family Aphididae) belonging to 12 genera, which have been reported to infest vegetable crops in J\&K are: Aphis craccivora Koch, Aphis fabae solanella Theobald, Aphis gossypii Glover, Aphis rumicis Linnaeus, Aphis spiraecola (Patch), Acyrthosiphon pisum Harris, Aulacorthum solani (Kaltenbach), Brachycaudus helichrysi (Kaltenbach), Brachycaudus rumexicolens (Patch), Brevicoryne brassicae (Linnaeus),
Cavariella aegopodii Scopoli, Hyadaphis coriandri (Das), Lipaphis (Lip.) erysimi (Kaltenbach), Lipaphis pseudobrassicae (Davis), Myzus persicae (Sulzer), Macrosiphum euphorbiae (Thomas), Semiaphis heraclei (Takahashi), Protrama penecaea Stryon

Summary of insect predator species (natural bio-control agents) of aphid pests on vegetable crops in J\&K

The 29 species of insect predators distributed over 25 genera, which have been reported on aphid pests in vegetable ecosystems in $\mathrm{J} \& \mathrm{~K}$, include 12 species of Coccinellidae (Coleoptera), 10 species of Syrphidae (Diptera), 2 species of Cecidomyiidae (Diptera), 3 Species of Chrysoperilidae (Neuroptera) and 2 species of Anthocoridae (Hemiptera) and are highlighted as under:-

\section{Order-coeloptera (family coccinellidae)}

Adalia tetraspilota (Hope), Calvia punctata (Mul.), Coccinella septempunctata L., Coccinella undecempunctata Linnaeus, Cheilomenes sexmaculatus Fabricius, Hippodamia variegata (Goe.), Harmonia dimidiata (Fabricius), Oenopia conglobata (Goe.), Platynaspidius saundersi (Crotch) (= Platynaspis saundersi), Priscibrumus uropygialis (=Exochomus uropygialis) (Mulsant), Propylea luteopustulata (Mulsant), Scymnus sp.

\section{Order-diptera (family syrphidae)}

Betasyrphus serarius (Wiedemann), Episyrphus balteatus DeGeer, Eupeodes (Macrosyrphus) confrater (Wiedem ann), Ischiodon scutellaris (Fabricius), Melanostoma univitatum (Wiedemann), Metasyrphus corolla (Fabr.), Paragus tibialis (Fallén, 1817), Sphaerophoria scripta (Linnaeus), Syrphus confractor Weid, 
(=Metasyrphus confractor), Syrphus sp.,

Order- diptera (family cecidomyiidae)

Aphidoletes aphidimyza (Rondani), Leucopis sp.

Order- Hemiptera (Anthocoridae): Anthocoris sp., Orius sp.

\section{Order-neuroptera (family chrysopidae)}

Chrysoperla zastrowi Sillemi (EsbenPetersen), Chrysoperla (=Chrysopa) carnea Stephens, $\quad$ Chrysoperla $(=$ Chrysopa $)$ orestes Banks

Summary of parasitoid species (natural bio-control agents) of aphid pests on vegetable crops in $J \& K$ region

The 17 species of aphid parasitoids (Hymenoptera: Braconidae: Aphidiinae) belonging to 7 genera reported on aphid pests of vegetable crops in J\&K are: Aphidius colemani Viereck, Aphidius eglanteriae Haliday, Aphidius matricariae Haliday, Aphidius salicis Haliday, Aphidius smithi Sharma and Subbarao, Aphidius sp., Diaeretiella rapae (McIntosh), Ephedrus persicae Froggatt, Ephedrus plagiator (Nees), Lysaphidus erysimi Stray, Lysiphlebus(Phelbus) fabarum (Marshall), Toxares zakai Shujauddin, Trioxys kahmirensis (Takada), Trioxys (Binodoxys) jaii, Bhagat, Trioxys (Binodoxys) indicus, Trioxys rubicola Shujauddin, Trioxys (Trioxys) complanatus Quilis

\section{Check list of family aphididae on vegetable} crops in J\&K

The 18 species of Aphids (Family Aphididae), which have been reported on vegetable crops in $J \& K$ are appended in the following checklist. The Aphid species in this checklist are documented subfamily-wise, \& systematically, with details given on their host-plant range (followed by references), insect predators (family wise and followed by references) and parasitoids (followed by references).

Sub-family: aphidinae

Tribe-aphidini Latreille, 1802

Aphis craccivora koch, 1854

Aphis craccivora is commonly known as cow pea aphid.

Host vegetable plants in $\mathrm{J} \& \mathrm{~K} /$ references

Cucumis sativus, Dolichos lablab, Lycopersicum esculentum, Phaseolus vulgaris, Rumex acetosa, Rumex nepalensis, Solanum melongena, Solanum tuberosum / (Bhagat, 2012; Bhat, 2017; Bhat and Bhagat, 2017; Khan et al., 2017; Bhat and Ahanger, 2018)

Predatory natural enemies in $J \& K /$ references

\section{Coleoptera (Coccinellidae)}

Adalia tetraspilota, Cheilomenes sexmaculata, Hippodamia variegata, Platynaspidius saundersi $\quad(=$ Platynaspis saundersi), Scymnus sp., Oenopia conglobata, Propylea luteopustulata;

\section{Diptera (Syrphidae)}

Eupeodes (Macrosyrphus) confrater, Ischiodon scutellaris, Melanostoma univitatum, Sphaerophoria scripta, Paragus tibialis / (Bhat, 2017; Khan et al., 2017; Bhat and Bhagat, 2017) 
Parasitoid natural enemies in $J \& K /$ references

(Hymenoptera: Braconidae: Aphidiinae): Diaeretiella rapae / (Bhagat \& Ahmad, 1991; Bhagat, 2008); Trioxys (Binodoxys) indicus / (Bhagat, 2008), Ephedrus persicae Froggatt, Trioxys jaii Bhagat/ (Stary and Bhagat, 1978; Bhagat, 1982 a, 1982b; Khan et al., 2017)

\section{Aphis fabae solanella Theobald, 1914}

It is commonly known as Black bean aphid.

Host vegetable plants in $\mathrm{J} \& \mathrm{~K} /$ references

Phaseolus sp. (French beans), Rumex acetosella, Rumex nepalensis / (Bhat \& Lone, 1984; Bhat, 2017; Khan et al., 2017; Bhat and Ahanger, 2018)

Predatory natural enemies in $J \& K /$ references

\section{Coleoptera (coccinellidae)}

Adalia tetraspilota, Coccinella septempunctata, Chrysoperla z. sillemi, Hippodamia dimidiate, Hippodamia variegate, Oenopia conglobata (Goe.); Diptera: (Chamaemyiidae): Aphodoletes aphidomyzae, Leucopis sp. /(Bhagat and Lone, 1084; Bhagat and Matta, 2002; Bhat,2017; Khan et al., 2017); Hemiptera (Anthocoridae): Anthocoris sp., Orius sp.

Parasitoid natural enemies in $J \& K /$ references

(Hymenoptera: Braconidae: Aphidiinae): Aphidius matricariae Haliday (Bhagat and Ahmad, 1991; Bhat et al., 2017); Lysiphelebus (Phelbus) fabrum, Trioxys (Binodoxys) indicus (Bhagat. 2008)

\section{Aphis gossypii Glover, 1802}

Aphis gossypii is commonly known as cotton aphid.
Host vegetable plants in $\mathrm{J} \& \mathrm{~K} /$ references

Capsicum annum, Cucumis sativus, Cucurbita maxima, Cucurbita pepo, Lagenaria siceraria, Rumex sp., Solanum melongena, S. tuberosum, Taraxacum officinale (Bhagat and Masoodi, 1986; Bhat et al., 2011; Khan et al., 2017; Bhat,2017 ; Bhat and Bhagat, 2017; Bhat \& Ahanger, 2018).

Predatory natural enemies in $J \& K /$ references

\section{Coeloptera (Coccinellidae)}

Adalia tetraspilota (Hope), Coccinella septempunctata L., Cheilomenes sexmaculatus, Hippodamia variegata (Goe.), Symnus sp., Priscibrumus uropygialis (=Exochomus uropygialis), Propylea luteopustulata (Mulsant); Neuroptera (Chrysopidae): Chrysoperla sillemi (EsbenPetersen), Chrysopa arestes; Diptera (Syrphidae): Episyrphus balteatus DeGeer (Ahmad and Bhat, 1986; Bhat, 2017; Bhat and Bhagat, 2017; Khan et el., 2017); Hemiptera (Anthocoridae): Anthocoris sp., Orius sp. (new records)

Parasitoid natural enemies in J\&K/ references

(Hymenoptera: Braconidae: Aphidiinae): Ephedrus plagiator (Nees), Trioxy srubicola Shujauddin (Rishi, 1976; Shujauddin, 1973; Khan et al., 2017)

\section{Aphis rumicis Linnaeus, 1758}

Host vegetable plants in $\mathrm{J} \& \mathrm{~K} /$ references

Rumix sp. (Khan et al., 2017)

Natural enemies and authors reporting

Predatory natural enemies in $J \& K /$ references

Coeloptera (Coccinellidae)

Adalia tetraspilota (Hope), Hippodamia variegate (Goe.), Propylea luteopustulata (Mul.) (Khan et al., 2017). 
Parasitoid natural enemies in $J \& K /$ references: not known

Aphis spiraecola (Patch, 1914)

Aphis spiraecola is commonly known as spirea aphid.

Host vegetable plants in $\mathrm{J} \& \mathrm{~K} /$ references

Cucumis sativus (Khan et al., 2017)

Natural enemies and authors reporting

Predatory natural enemies in $J \& K /$ references

Coeloptera (Coccinellidae)

Adalia tetraspilota, Coccinella septempunctata, $\mathrm{H}$. dimidiata, Hippodamia variegata (Goe.); Neuroptera (Chrysopidae): Chrysoperla z. sillemi (Khan et al., 2017)

Parasitoid natural enemies in $J \& K /$ references

(Hymenoptera: Braconidae: Aphidiinae): Aphidius matricariae Haliday (Stary and Bhagat, 1978; Khan et al., 2017)

Tribe-Macrosiphini Wilson, 1910

Acyrthosiphon pisum Harris, 1776

Host vegetable plants in $\mathrm{J} \& \mathrm{~K} /$ references

Phaseolus sp., Pisum sativum Linn (Ahmad and Bhat, 1986; Bhagat, 1986, 2012; Bhat et al., 2011; Khan et al., 2017; Bhat and Ahanger, 2018)

Predatory natural enemies in $J \& K /$ references

\section{Coeloptera (Coccinellidae)}

Adalia tetraspilota (Hope), Hippodamia variegate (Goe.); Diptera (Syrphidae):
Episyrphus balteatus, Melanostoma univitatum (Ahmad and Bhat, 1986; Bhat et al., 2011; Bhat and Bhagat, 2017)

Parasitoid natural enemies in $J \& K /$ references

(Hymenoptera: Braconidae: Aphidiinae): Aphidius eglanteriae (Bhagat, 2008); Aphidius smithi Sharma and Subbarao (Takada and Rishi, 1980); Trioxys Trioxys complanatus Quilis (Bhagat, 2008).

Aulacorthum solani (Kaltenbach, 1843)

Host vegetable plants in $\mathrm{J} \& \mathrm{~K} /$ references

Hibiscus esculentus (Okra) (khan et al., 2017)

Predatory natural enemies in $J \& K /$ references

Coeloptera (Coccinellidae)

Adalia tetraspilota (Hope), Coccinella septempunctata, Hippodamia variegata (Goe.) (khan et al., 2017).

Parasitoid natural enemies in $J \& K /$ references

(Hymenoptera: Braconidae: Aphidiinae): Aphidius sp. (khan et al., 2017).

Brachycaudus helichrysi (Kaltenbach, 1843)

Brachycaudus helichrysi is commonly known as green peach aphid.

Host vegetable plants in $\mathrm{J} \& \mathrm{~K} /$ references

Brassica oleracea (Khan et al., 2017)

Predatory natural enemies in $J \& K /$ references

Coeloptera (Coccinellidae)

Adalia tetraspilota (Hope), Coccinella 
septempunctata L., Hippodamia variegata (Goe.), Propylea luteopustulata; Neuroptera (Chrysopidae): Chrysoperla sillemi E. \& P. (Khan et al., 2017)

Parasitoid natural enemies in $J \& K /$ references

(Hymenoptera: Braconidae: Aphidiinae): Aphidius matricariae Haliday (Khan et al., 2017)

Brachycaudus rumexicolens (Patch, 1917)

Host vegetable plants in $\mathrm{J} \& \mathrm{~K} /$ references

Rumex sp. (Khan et al., 2017)

Predatory natural enemies in $\mathbf{J \& K} /$ references

\section{Coeloptera (Coccinellidae)}

Adalia tetraspilota, Calvia punctata (Mul.), Hippodamia variegata (Goe.); Neuroptera (Chrysopidae): Chrysoperla sillemi E. \& P. (Khan et al., 2017)

Parasitoid natural enemies in J\&K/ references: not known

Brevicoryne brassicae (Linnaeus, 1758)

It is commonly known as cabbage aphid.

\section{Host vegetable plants in $\mathrm{J} \& \mathrm{~K} /$ references}

Brassica oleracea (Linn.), Brassica oleracea var. acephella, Brassica o. var. botrytis, $B$. oleracea var. capitata, B. o.var. kashmiriana, Brassica oleracea var. gongylodes (Ahmad and Bhat, 1986; Bhagat, 1986, 2012; Zaz, 2001; Pandey et al., 2006; Khan et al., 2009, 2017; Bhat et al., 2011; Bhat, 2017; Bhat \& Bhagat, 2017; Bhat and Ahanger 2018).
Predatory natural enemies in $\mathbf{J} \& \mathbf{K} /$ references

\section{Coeloptera (Coccinellidae)}

Adalia tetraspilota (Hope), Cheilomenes sexmaculata, Coccinella septempunctata, Hippodamia variegate (Goe.), Propylea luteopustulata (Mul.); Diptera (Syrphidae): Episyrphus balteatus, Sphaerophoria scripta (Bhgat and Lone, 1984; Ahmad and Bhat, 1986; Khan et al., 2009,2017; Bhat, 2017; Bhat and Bhagat, 2017; Khan et al., 2017)

Parasitoid natural enemies in $J \& K /$ references

(Hymenoptera: Braconidae: Aphidiinae): Aphidius matricariae Haliday, Diaeretiella rapae (M'Int) (Rao et al., 1969; Bhagat and Amad, 1991; Stary \& Bhagat, 1978; Bhagat, 1986; Bhat, 2008; Bhat et al., 2017; Bhagat and Ahmad, 1991)

\section{Cavariella aegopodii Scopoli 1763}

Host vegetable plants in $\mathrm{J} \& \mathrm{~K} /$ references

Daucus carota (Bhat, 2017; Bhat and Ahanger, 2018)

Predatory natural enemies in J\&K/ references

Diptera (Syrphidae): Episyrphus balteatus (Bhat and Bhagat, 2017)

Parasitoid natural enemies in $J \& K /$ references: Not known

Hyadaphis coriandri (Das, 1918)

Host vegetable plants in $\mathrm{J} \& \mathrm{~K} /$ references

Coriandrum sativum (Corriander) (Khan et al., 2017) 
Predatory natural enemies in $J \& K /$ references

\section{Coeloptera (Coccinellidae)}

Adalia tetraspilota (Hope), Calvia punctata (Mul.), Coccinella septempunctata L, Hippodamia variegata (Goe.); Neuroptera (Chrysopidae): Chrysoperla z. sillemi E. \& P. (Khan et al., 2017)

Parasitoid natural enemies in $J \& K /$ references

(Hymenoptera: Braconidae: Aphidiinae): Aphidius matricariae H. (Khan et al., 2017)

Lipaphis (Lip.) erysimi (Kaltenbach, 1843)

Host vegetable plants in $\mathrm{J} \& \mathrm{~K} /$ references

Brassica napus (Linn.) $\quad(=$ Brassica campestris L.), Brassica campestris), Brassica napus (Bhagat, 1986; Bhat et al., 2011; Bhagat, 2012; Bhat and Ahanger, 2018)

Predatory natural enemies in $J \& K /$ references: Coeloptera (Coccinellidae)

Adalia tetraspilota (Hope), Calvia punctata, Cheilomenes sexmaculata, Coccinella septempunctata L., $\quad$ Coccinella undecempunctata, Hippodamia variegate (Goe.), Oenopia congoblata; Diptera (Syrphidae): Sphaerophoria scripta, Melanostoma univitiatum, Ischiodon scutellaris, Metasyrphuscorolae, Metasyrphus confractor, Paragus seratus, Betasyrphus serarius, Syrphus sp. (Bhagat and Lone, 1984; Ahmad and Bhat, 1986; Bali, 1987; Bhagat and Matta, 2002; Khan et al., 2009; Bhat, 2017; Bhat and Bhagat, 2017)
Parasitoid natural enemies in $J \& K /$ references

(Hymenoptera: Braconidae: Aphidiinae): Diaertiella rapae (Rao et al., 1969; Rishi, 1976; Bhagat, 1986; Bhagat and Ahmad, 199; Bhat et al., 2017)

Lipaphis pseudobrassicae (Davis, 1914)

\section{Host vegetable plants in $\mathrm{J} \& \mathrm{~K} /$ references}

Brassica oleracea Brassica juncaea Brassica oleracea var capitata, B. rapa, Raphanus sativa (Khan et al., 2017)

Predatory natural enemies in $J \& K /$ references: Coeloptera (Coccinellidae):

Adalia tetraspilota (Hope), Coccinella septempunctata L., Propylea luteopustulata (M.), Hippodamia variegate (Goe.); Neuroptera (Chrysopidae): Chrysoperla sillemi E.\& P. (Khan et al., 2017)

Parasitoid natural enemies in $J \& K /$ references

(Hymenoptera: Braconidae: Aphidiinae): Lysaphidus erysimi Stray (Khan et al., 2017)

Myzus persicae (Sulzer, 1762)

Myzus persicae is commonly known as green peach aphid.

\section{Host vegetable plants in $\mathrm{J} \& \mathrm{~K} /$ references}

Capcicum annuи, Cucurbita maxima Lycopersicon esculentum Solanum melongena, Solanum tuberosum (Bhat, et al., 2011; Bhagat, 2012; Khan et al., 2017; Bhat and Ahanger, 2018) 
Predatory natural enemies in $J \& K /$ references

\section{Coeleoptera (Coccinellidae)}

Adalia tetraspilota (Hope), Cheilomenes sexmaculata, Coccinella septumpunctata, Hippodamia variegate (Goe.), Propylea luteopustulata (Mul.), Scymnus sp.; Diptera (Syrphidae): Episyrphus balteatus (Bhat, 2017; Bhat and Bhagat, 2017; Khan et al., 2017); Hemiptera (Anthocoridae): Anthocoris sp., Orius sp. (new record) ; Neuroptera (Chrysopidae): Chrysopa (Chrysoperla carnea) Stephens (Bhat, 2008a)

Parasitoid natural enemies in $J \& K /$ references

(Hymenoptera: Braconidae: Aphidiinae): Aphidius matricariae Haliday (Bhagat, 2008), Aphidius colemani Viereck, Toxares zakai Shujauddin (Shujauddin, 1973)

Macrosiphum euphorbiae (Thomas, 1878)

Host vegetable plants in $J \& K /$ references

Solanum melongena (khan et al., 2017)

Predatory natural enemies in $J \& K /$ references

\section{Coeleoptera (Coccinellidae)}

Adalia tetraspilota (Hope), Coccinella septempunctata, Hippodamia variegata (Goe.) (khan et al., 2017); Hemiptera (Anthocoridae): Anthocoris sp. (new record)
Parasitoid natural enemies in $J \& K /$ references

(Hymenoptera: Braconidae: Aphidiinae): Trioxys kahmirensis (Takada) (Takada and Rishi, 1980; khan et al., 2017)

Semiaphis heraclei (Takahashi, 1921)

Host vegetable plants in $\mathrm{J} \& \mathrm{~K} /$ references

Daucus carota (Bhagat, 1986, 2012; Bhat and Ahanger,2018)

Predatory natural enemies in $\mathrm{J} \& \mathrm{~K} /$ references: Not known

Parasitoid natural enemies in J\&K/ references

(Hymenoptera: Braconidae: Aphidiinae): Aphidius salicis (Bhagat, 1986; Bhagat, 2008)

Sub-family-Lachinae Herrich-Schaeffer, 1854

The tribe- Tramini

Protrama penecaea Stryon 1998

Host vegetable plants in $\mathrm{J} \& \mathrm{~K} /$ references

Helianthus tuberosus (Artichoke) (Khan et al., 2017)

Predatory natural enemies in $J \& K /$ references

Coeleoptera (Coccinellidae): Adalia tetraspilota (Hope), Calvia punctata (Mul.), Hippodamia variegata (Goe.); Neuroptera (Chrysopidae): Chrysoperla z. Sillemi E. \& P. (Khan et al., 2017). Parasitoids / references: Not known. 
Table.1 Species richness of Aphids and their natural enemies on vegetable crops in J\&K

\begin{tabular}{|c|c|c|c|c|c|c|c|c|c|c|}
\hline \multirow{2}{*}{\multicolumn{3}{|c|}{ Aphids }} & \multicolumn{8}{|c|}{ Natural enemies } \\
\hline & & & \multicolumn{4}{|c|}{ Predators } & \multicolumn{4}{|c|}{ Parasitoids } \\
\hline 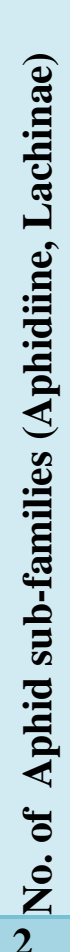 & 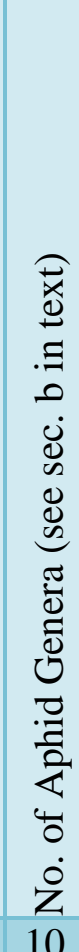 & 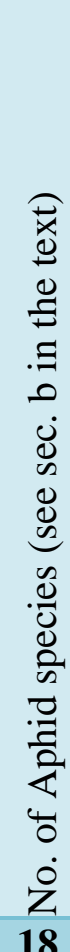 & 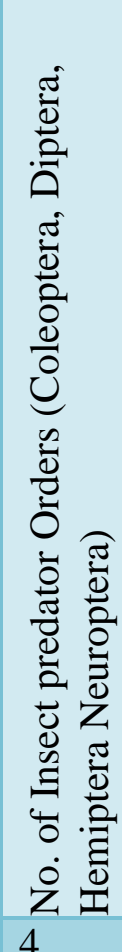 & 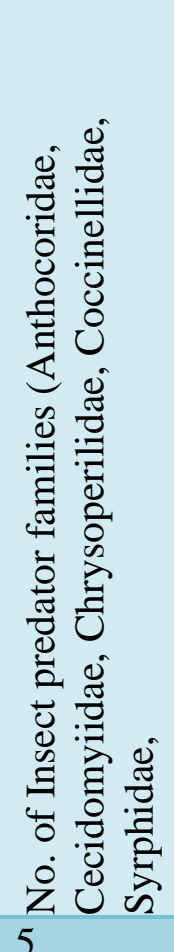 & 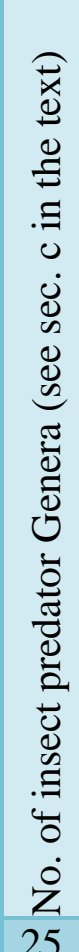 & 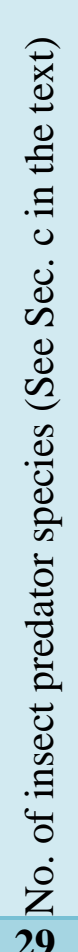 & 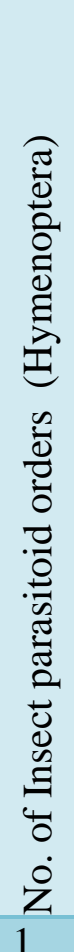 & 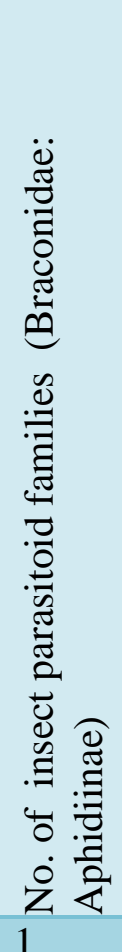 & 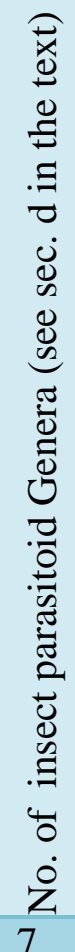 & 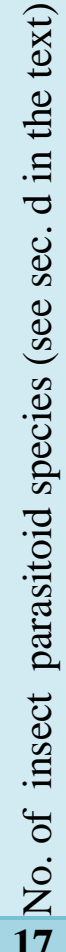 \\
\hline 2 & 10 & 18 & 4 & 5 & 25 & 29 & & 1 & & 17 \\
\hline
\end{tabular}

Legends to Figures (Photo credit Deen Mohd.)

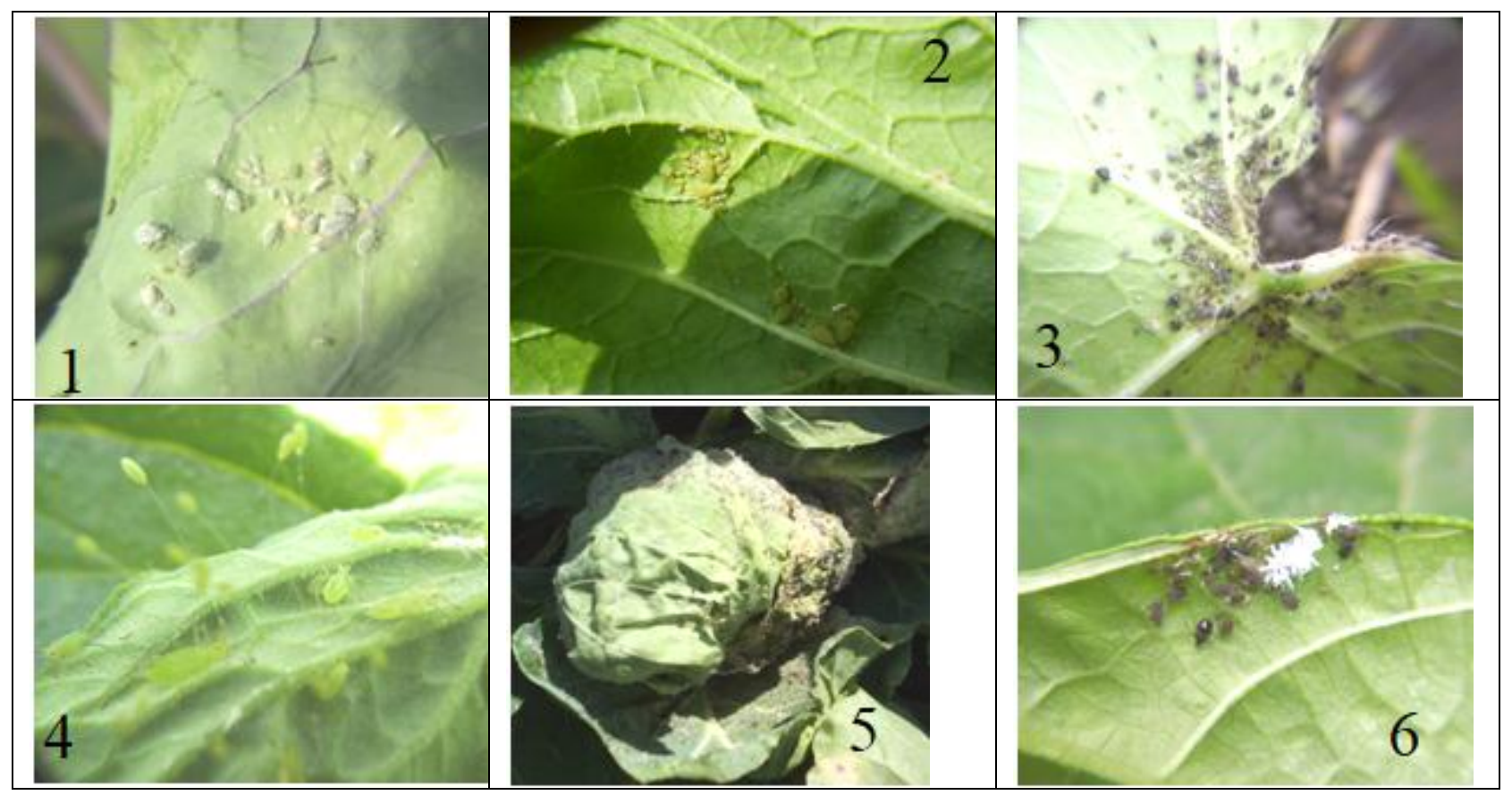




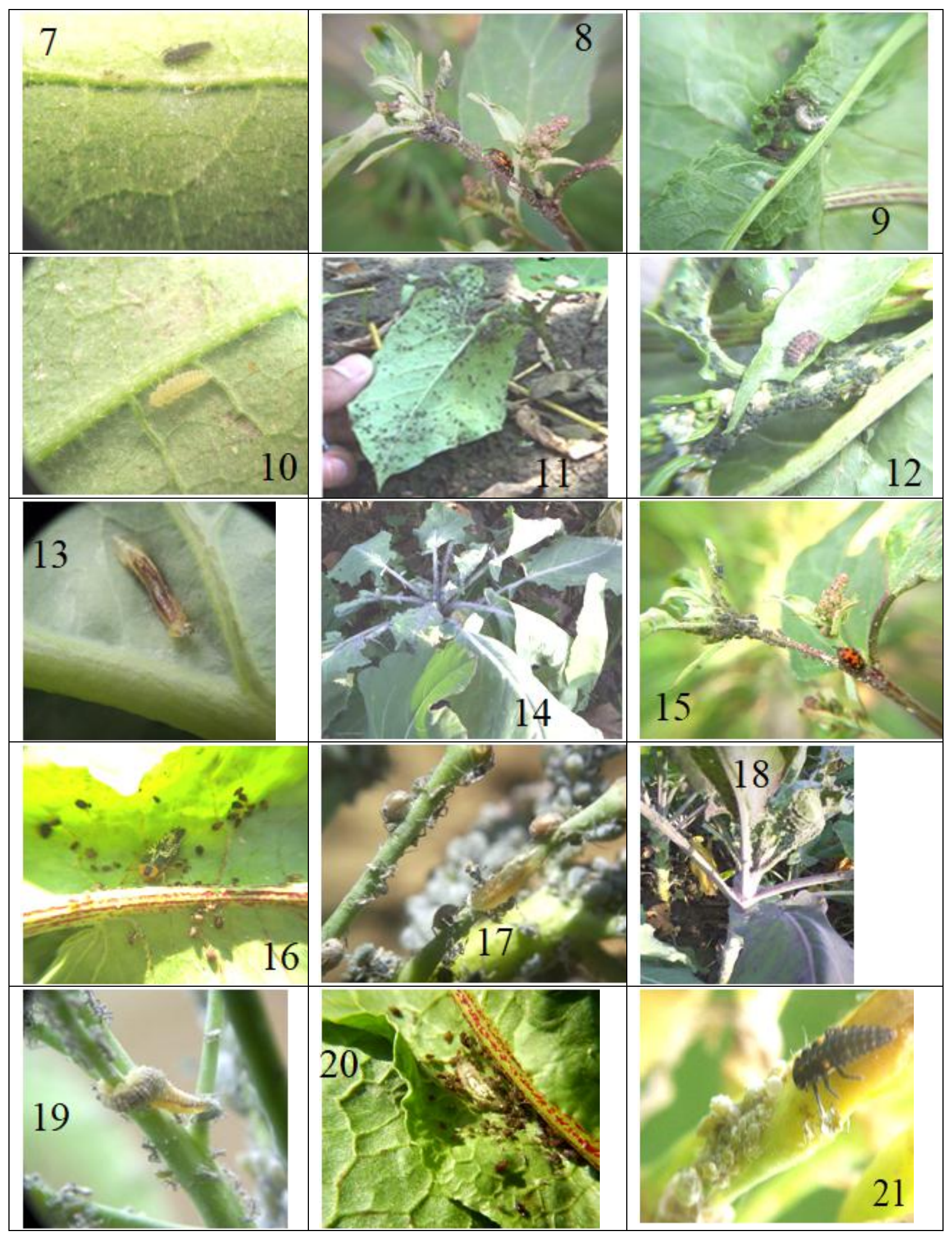




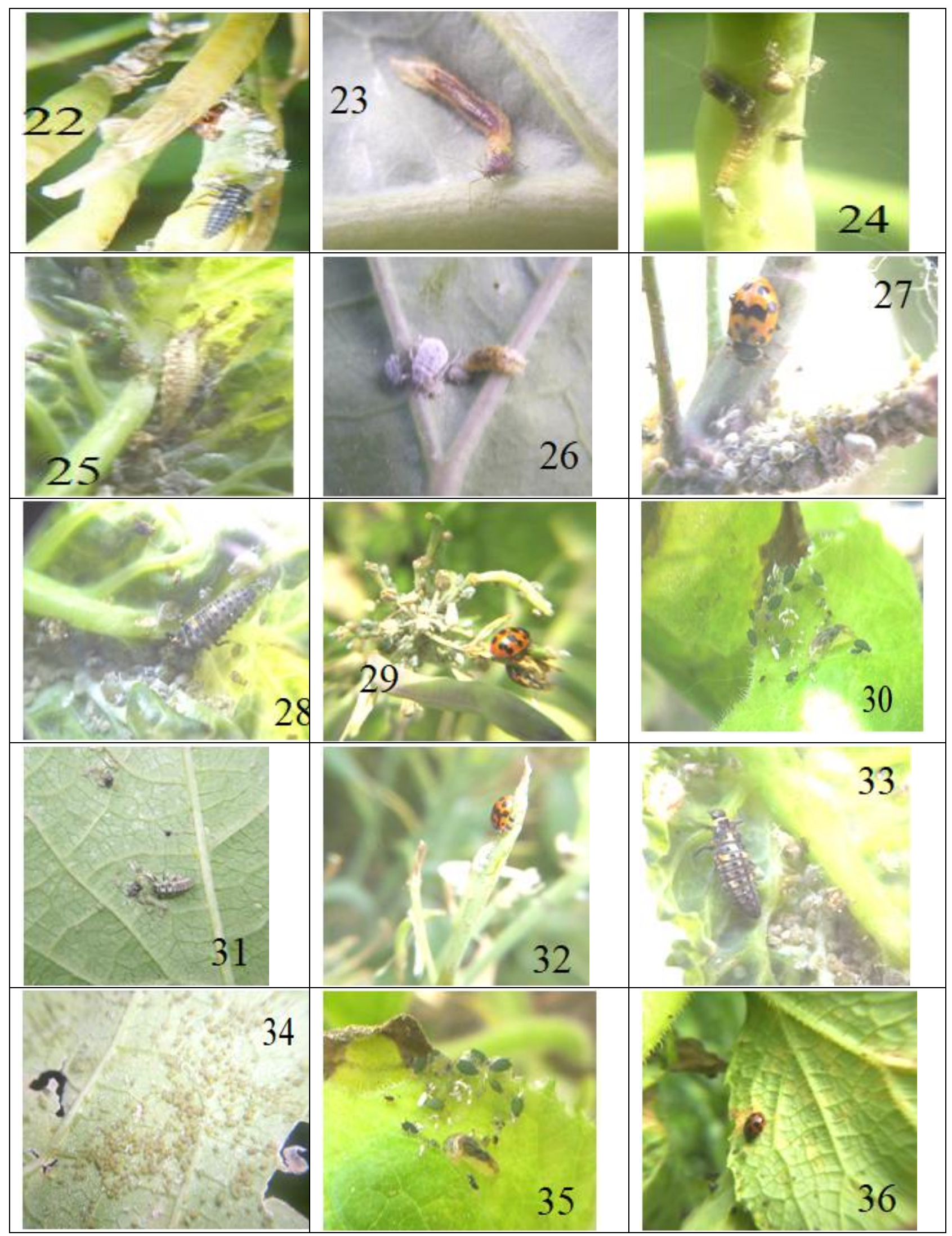




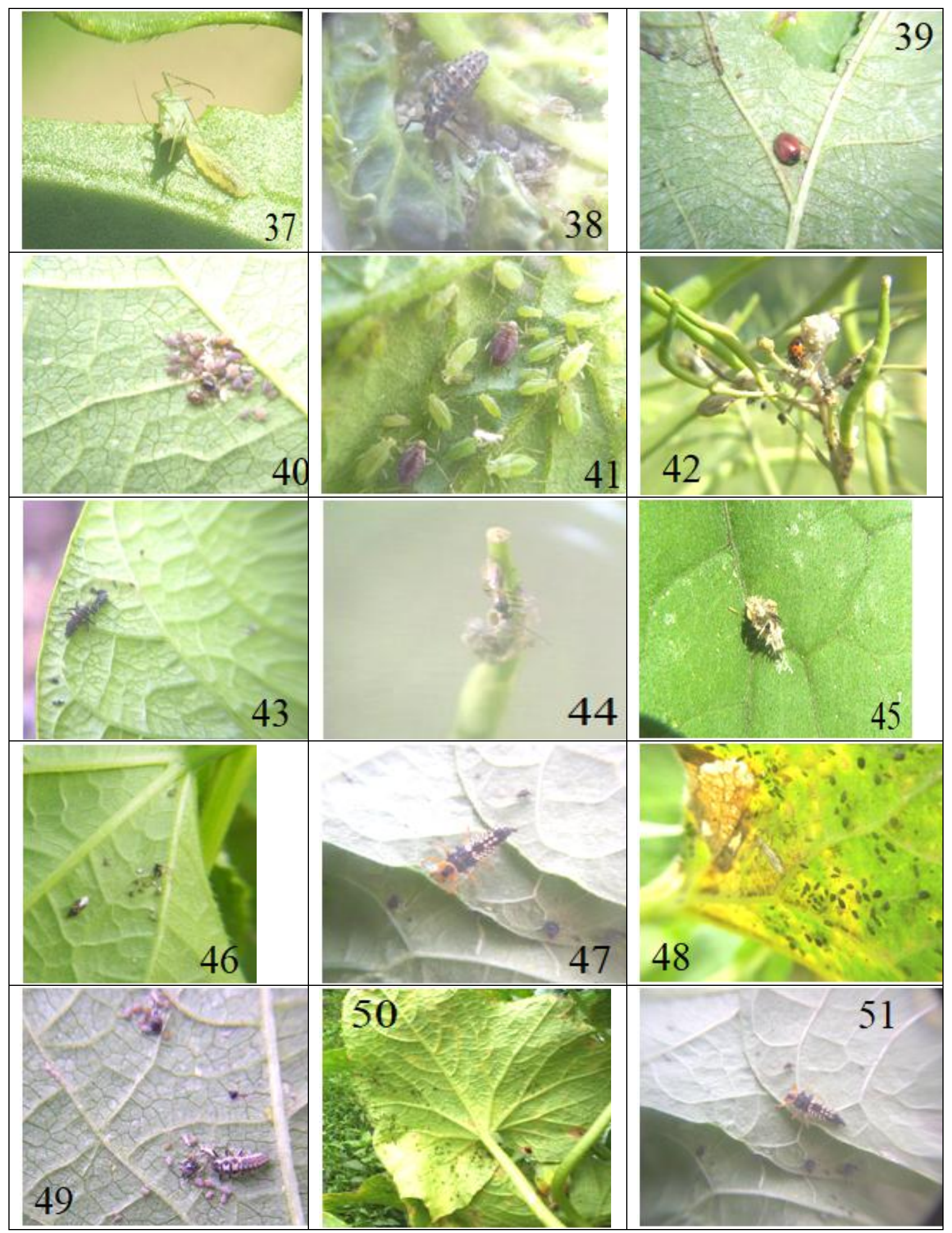




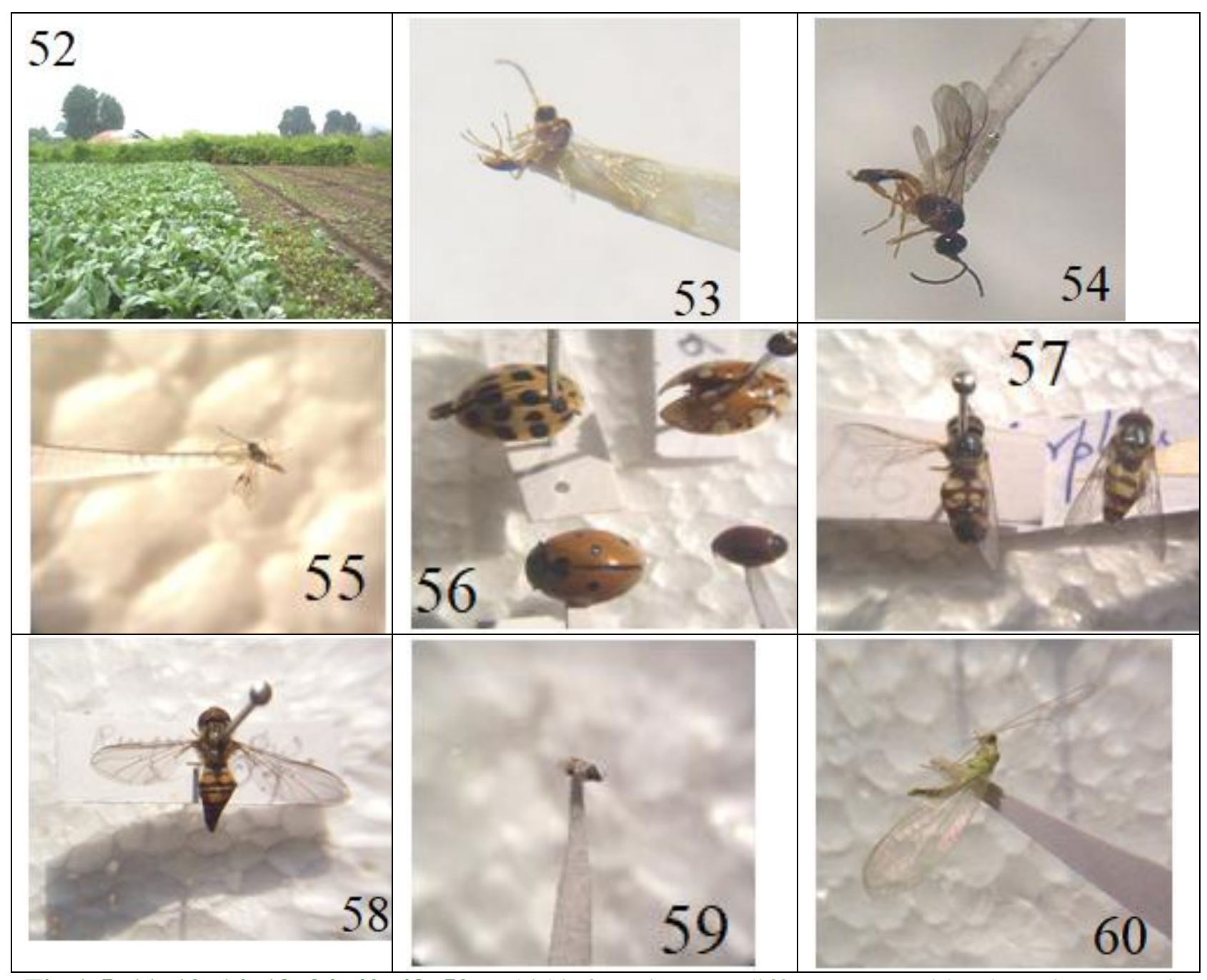

Fig.1-5, 11, 12, 14, 18, 34, 40, 48, 50 Aphid infestations on different vegetable plants in Kashmir Fig.6, 7, 9, 10, 16, 21, 22, 28, 31, 33, 38, 43, 45, 47, 49, 51 Larvae of coccinellid beetles (predators) feeding on aphid pests on different vegetable plants in Kashmir

Fig.13, 17, 19, 20, 23, 24, 26, 30, 35, 37 larvae of syrphid flies (predators) feeding on aphid pests on different vegetable plants in Kashmir

Fig.8, 15, 27, 29, 32, 36, 39, 42 adult coccinellid beetles (predators) feeding on aphid pests on different vegetable plants in Kashmir

Fig.25 larva of Chrysoperla feeding on aphids in vegetable fields in Kashmir

Fig.51 Predatory bug Anthocoris sp. recovered from vegetable fields while feeding on aphids in Kashmir

Fig.53 Parasitoid Trioxys sp. recovered from aphids in Kashmir

Fig.54-55 Parasitoid D. rapae recovered from aphid B. brassicae in Kashmir

Fig.57 Adult syrphid fly Metasyrphus sp. (Syrphidae), predator of aphids, collected after rearing of its larvae in Kashmir

Fig.60 Adult Chrysoperla recovered after rearing of its larvae on aphids in Kashmir 


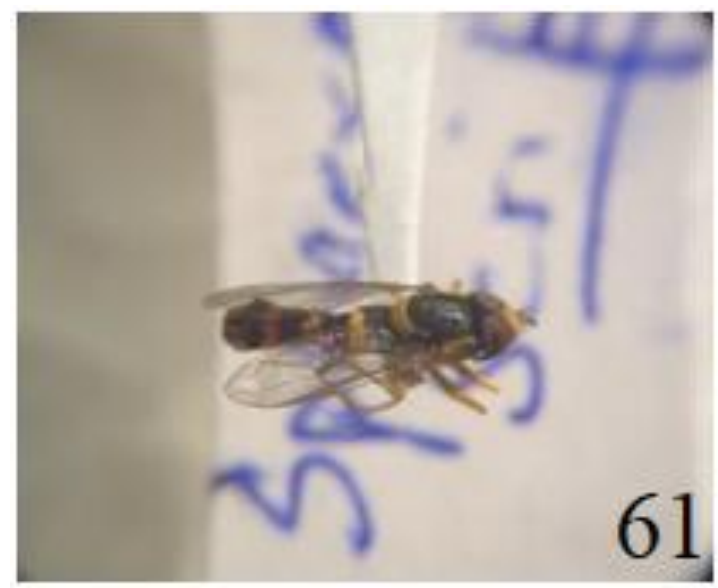

Fig.61 Adult syrphid fly Sphaerophoria scripta, predator of aphids, collected after rearing of its larvae in Kashmir

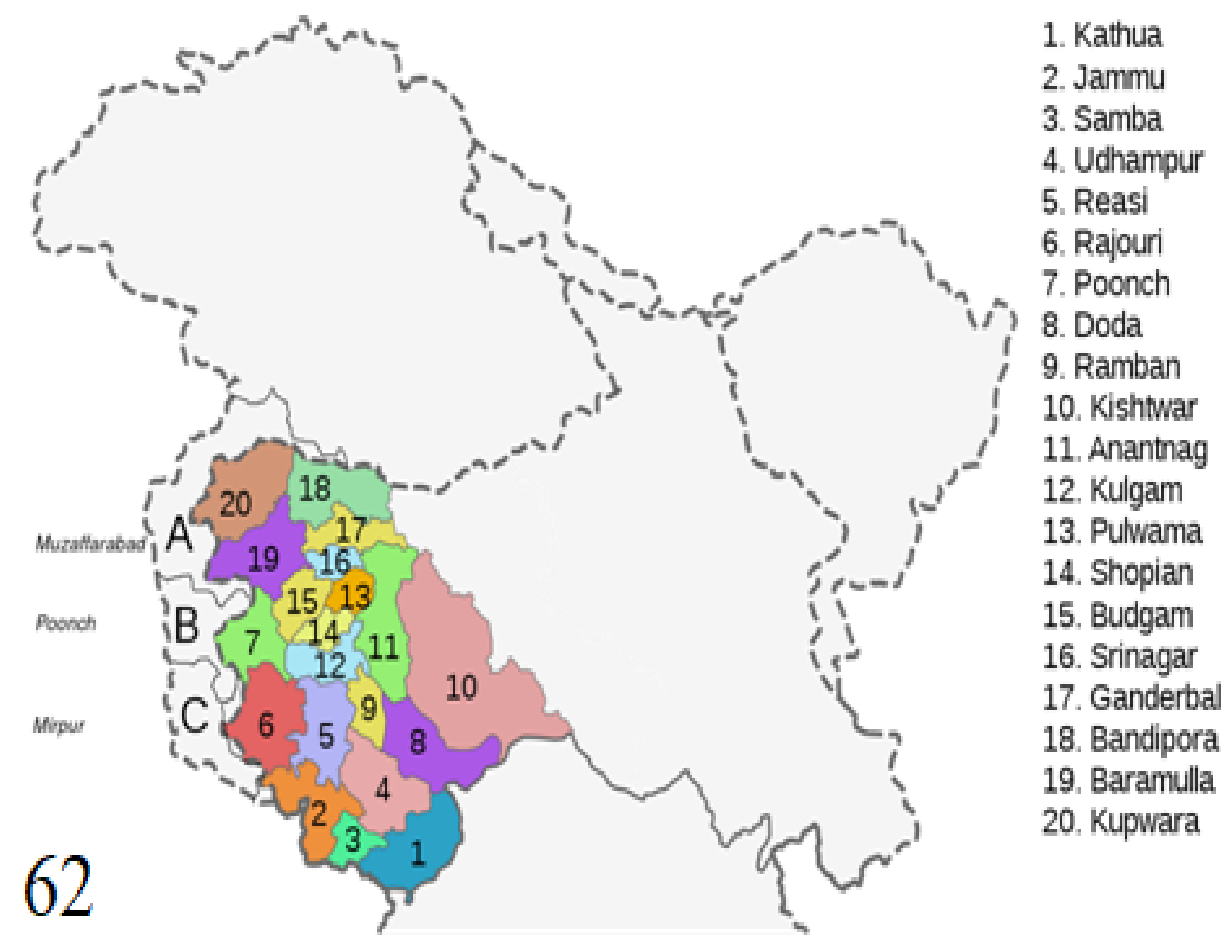

Fig.62 Map of J\&K showing study areas (Districts) in Kashmir

Since, the complete knowledge of aphids is crucial for understanding their pest nature and for formulating a proper control and management strategy in order to mitigate their damage on vegetable crop ecosystems, So, the present work, based on field and literature survey provided a base line data pertaining aphid pests and their natural bio-control agents in vegetable ecosystems in $\mathrm{J} \& \mathrm{~K}$. Based on this work, it is understood that there are large number of aphid species (at least 18) which attack various types of vegetable crops in this region.

However there are even more diverse kinds of natural bio-control agents of these aphid pests represented by 17 species of parasitoids and 29 species of predators, which mitigate the damage caused by aphids in vegetable ecosystems of this region. 
Thus, it is concluded that it is important to preserve and conserve the existing diversity of such natural enemies so that efforts of integrated control program, if planned for vegetable crop ecosystems in this region, can materialize and be successful.

\section{References}

Agarwala BK and Ghosh AK. 1984. A checklist of Aphidoidea (Homoptera) of India. Records of Zoological Survey of India. 50: 73.

Ahmad D and Bhat MR. 1986. Distribution and host range of some aphidophagous Syrphid flies in Kashmir. Geobios New Reports. 5: 165-166.

Bali R. 1987. Coccinellids (Coleoptera) of Kashmir Valley, distribution and host range. Geobios New Reports. 6: 187-89.

Bhagat KC and Masoodi MA. 1986. Record of green lacewing fly, Chrysopa arestes Banks (Chrysopidae: Neuroptera) as a predator of brinjal aphid, Aphis gossypii Glover (Aphididae: Homoptera). Indian Journal of Plant Protection. 13(2): 132.

Bhagat RC and Ahmad MN. 1991. Aphidiid parasitoids (Hymenoptera) of Aphids (Homoptera) of Jammu-new records, host range and biological notes. Journal of Aphidology. 5: 90-96.

Bhagat RC and Lone MA. 1984. New records and host range of predators of aphids (Aphididae: Homoptera) in Kashmir Valley, India. Science and Culture. 50(12):333-372

Bhagat RC and Matta A. 2002. Host range and diversity of aphidophagous insect (predators) of Kashmir Himalaya, with new host aphid/plant records, In: Khan, M. A. (Ed.). Environment Biodiversity and Conservation. A. P.H. Pub. New Delhi. Pp. 269-282

Bhagat RC. 1982a. New aphid parasitoids (Hymenoptera: Aphidiiae) from Kashmir, India. Oriental Insects. 16:113-117.

Bhagat RC. 1982b. On two new parasitoids
(Hymenoptera Aphidiiae) from Kashmir. Entomon. 7(3):321-324

Bhagat RC. 1986. On aphid pests and their aphidoid parasitoids of agricultural importance. Indian Agriculturists. 30 (3): 229-235

Bhagat RC. 2008. Biodiversity of parasite-fauna of Jammu, Kashmir and Ladakh. Paramount Publishing Corporation, New Delhi. Pp. 232

Bhagat RC. 2012. Aphids (Insecta) of agricultural importance in $\mathrm{J} \& \mathrm{~K}$ state, India: a checklist and biodiversity. International Journal of Food, Agriculture and Veterinary Science. 2(3): 116-125.

Bhat DM and Ahanger FA. 2018. A systematic checklist and species richness of insect pests associated with vegetable crops in Jammu \& Kashmir State (India). Journal of Entomology and Zoology studies 6 (2): $328-338$

Bhat DM and Bhagat RC. 2017. Host Range and Diversity of Syrphid Predators (Insecta: Diptera) of aphids on Vegetable Crops of Kashmir, with New Host Aphid/ Plant Records. Trends in Bioscinces. 10(6): 1446-1448

Bhat DM, Bhagat RC and Qureshi AA. 2011. A survey of insect pests damaging vegetables crops on Kashmir valley (India), with some new records. Journal of Entomological Reserach. 35(1):85-91.

Bhat DM, Bhagat RC and Qureshi AA. 2017. Parasitoid fauna associated with insect pests of vegetable crops of Kashmir Himalaya, India: check list and biodiversity. Munis Entomology and Zoology. 12(1): 168-174

Bhat DM. 2008a. Studies on insect parasites and predators of some insect pests of vegetable crops of Kashmir Valley. Ph.D thesis, P.G. Department of Zoology, University of Kashmir, Srinagar, J\&K (India).

Bhat DM. 2017. Host range and diversity of Coccinellid (Coleoptera) predators of aphid pests in vegetable crop ecosystems of Kashmir, with new host aphid/plant 
records. Journal of Entomological Research. 41 (2): 183-186

Bhat MA. 2008. A report on insect pests associated with Cole crops in Kashmir. Applied Biological Research. 10:66-67

Blackman RL and Eastop VF. 2006. Aphids on the World's Herbaceous Plants and Shrubs. Wiley, Chichester. Pp. 1024

Blackman RL and Eastop VF. 2007. Taxonomic Issues. In: Aphids as Crop Pests. (Eds. H.F. van Emden and R. Harrington), CABI, Cambridge, UK. Pp. 1-22

Dixon AFG. 1987. Parthenogenetic reproduction and the rate of increase in aphids. In: Aphids; their Biology, Natural Enemies and Control, (Eds. A.K. Minks and P. Harrewijin), Elsevier, Amsterdam. Pp. 269-287

Dixon AFG. 1998. Aphid Ecology, 2nd edn., Chapman and Hall, London. Pp. 300

Ghosh AK and Ghosh LK. 2006. The Fauna of India and Adjacent Countries: Homoptera, Aphidoidea, Zooogical Survey of India, Calcutta.7 (1): 244.

Khan AA, Shah MA and Riyaz S. 2017. Records of aphid and their natural enemies in agro-ecosystem with special reference to horticultural ecosystem of Kashmir. Journal of Entomology and Zoology Studies. 5(4): 189-203

Khan AA, Zaki FA, Khan ZH and Mir MA. 2009. Biodiversity of predaceous beetles (Coleoptera: Coccinellidae) in Kashmir. Journal of Biological Control. 23: 43-47.

Pandey AK, Namgyal D, Mehdi M, Mir MS and Shiekh BA. 2006. A case study: Major insect pests associated with different vegetable crops in cold and region Ladakh, of Jammu and Kashmir. Journal of Entomological Research. 30(2):169-174

Rao VP, Ramaseshiah G, Sethumadhavan TV and Ramaswamy P. 1969. Study of natural enemies of aphids (for the U. S. A.). Report of the Commonwealth Institute of Biological Control. Pp: 45-47.

Remaudiere G and Remaudiere M. 1997. Catalogue of World's Aphididae: Homoptera- Aphidoidea. Inra Edition, Route de France. Pp. 478

Shah MA and Khan AA. 2013. Aphid in agroecosystem and their management. In: Hill Agriculture: Prospects, Constraints and Mitigations, edited by Dr. Shahid Ahamad, Daya Publishing House, New Delhi. Pp. 314-330

Shah MA, Khan AA, Junaid JM, Majid S and Mohi-ud-din S. 2015. Aphid Vectored Viral Diseases and their Management. In: Insect Pests Management of Fruit Crops, Edited by Ajay Kumar Pandey, Dr. Pramod Mall, paper 27, Biotech Books, Ansari Road, Darya Ganj, New Delhi. Pp. 511-554.

Shujauddin. 1973. Three species of Trioys Haliday (Hymenoptera Aphidiiae) from India. Indian Journal of Entomology. 35: 9-14

Stary P and Bhagat RC. 1978. Records and descriptions of aphid parasitoids from Kashmir, India (Hymenoptera: Aphidiidae). Acta Entmologica Bohemoslov. 75: 387-393

Takada H and Rishi ND. 1980. Records of fifteen species of Aphidiiae (Hymenoptera) from Kashmir, India with descriptions of three new species. Kontyu. 48:234240.

Zaz GM. 2001. Incidence and population buildup of cabbage aphid, Brevicoryne brassicae on cabbage and cauliflower. Applied Biological Research. 3 ( 1 \& 2): 51-53

\section{How to cite this article:}

Deen Mohd. Bhat, Sajad Ahmad Khan, Fayaz Ahmad Ahanger and Muzafar Ahmad Sheikh. 2020. Diversity of Aphid Pests (Homoptera: Aphididae) and their Natural Bio-Control Agents in Vegetable Crop Ecosystems of Jammu \& Kashmir, (India). Int.J.Curr.Microbiol.App.Sci. 9(05): 2529-2546. doi: https://doi.org/10.20546/ijcmas.2020.905.290 